\title{
The Comparative Effects of Gene Modulators on Thyroid-Specific Genes and Radioiodine Uptake
}

\author{
Murat Tuncel, ${ }^{1}$ Didem Aydin, ${ }^{2}$ Elif Yaman, ${ }^{3}$ Uygar H. Tazebay, ${ }^{3}$ Dicle Güç, ${ }^{2}$ A. Lale Doğan, ${ }^{2}$ \\ Burçin Taşbasan, ${ }^{2}$ and Ömer Uğur ${ }^{1}$ \\ ${ }^{1}$ Department of Nuclear Medicine, Hacettepe University Faculty of Medicine, Ankara, Turkey \\ ${ }^{2}$ Department of Basic Oncology, Hacettepe University Oncology Institute, Ankara, Turkey \\ ${ }^{3}$ Department of Molecular Biology and Genetics, Bilkent University, Ankara, Turkey
}

\begin{abstract}
The aim of this study was to comparatively investigate the effects of 5-azacytidine-C (5-Aza), trichostatinA (TSA), and all-trans retinoic acid (ATRA) on mRNA expressions of Na/I symporter (NIS), thyroglobulin $(T g)$, thyroid peroxidase (TPO), and thyroid stimulating hormone receptor (TSH-R), and radioiodine (RAI) uptake in cancer (B-CPAP) and normal (Nthy-ori 3-1) thyroid cell lines. Cell lines were treated with $10 \mathrm{ng} / \mathrm{mL}$ of TSA, $5 \mu \mathrm{M}$ of 5-Aza, and $1 \mu \mathrm{M}$ of ATRA, according to the MTT (methyl-thiazol-tetrazolium) test results. Additionally, recombinant thyroid stimulating hormone (rTSH) was also applied, with a selected dose of $100 \mathrm{ng} / \mathrm{mL}$. Following the treatment, NIS, Tg, TPO, and TSH-R mRNA levels were detected by real-time-polymerase chain reaction (RT-PCR) and RAI uptakes were measured by using a well counter as the counts/cell number. 5-Aza increased TSH-R mRNA expression in both of the cell lines and decreased TPO, NIS, and Tg mRNA levels in the cancer cell line. In the normal thyroid cell line, 5-Aza increased TPO mRNA levels 2-fold and made no differences in NIS and Tg mRNA levels. TSA treatment repressed NIS and Tg mRNA levels, and made no differences on other thyroid specific genes investigated in the cancer cell line. In the normal thyroid cell line, TSA increased TSH-R mRNA levels in 72 hours and created no important differences in other genes. ATRA repressed the TSH-R mRNA levels in the normal thyroid cell line and increased the TPO and Tg mRNA levels slightly in both cell lines. Furthermore, in short-term treatment, ATRA repressed NIS gene expression slightly, but in the long term, this repression turned to basal levels. 5-Aza, TSA, and ATRA did not make any differences in RAI uptake in the cancer cell line, but rTSH increased RAI uptake significantly. In the normal thyroid cell line, TSA and ATRA decreased RAI uptake (to $1 / 10$ and $1 / 2$, respectively), but 5-Aza and rTSH increased RAI uptake significantly (2- and 4-fold, respectively). We have shown an increase in TSH-R gene expression and radioiodine uptake with 5-Aza. Further in vitro and in vivo studies are needed to support our findings and the potential clinical use of this agent.
\end{abstract}

Key words: 5-azacytidine-c, trichostatin A, TPO, NIS, mRNA

Address reprint requests to: Ömer Ugur; Department of Nuclear Medicine, Hacettepe University Faculty of Medicine, TR-06100, Sihhiye, Ankara, Turkey; Tel.: +90-3123051336; Fax: +90-312-3093508

E-mail: ougur@hacettepe.edu.tr

\section{INTRODUCTION}

The main therapy modalities in the treatment of the patients suffering from thyroid cancer are surgery and radioiodine (RAI) therapy. ${ }^{1,2}$ The nor- 
mal thyroid cells have certain specific properties, such as RAI uptake, sodium iodine symporter (NIS), thyroid peroxidase (TPO) activity, thyroglobulin (Tg) synthesis, and thyroid-stimulating hormone receptors (TSH-Rs). During the malign dedifferentiation process, these properties were lost, which led to a problematic patient group who had residual thyroid cancer with elevated $\mathrm{Tg}$ levels but negative I-131 scintigraphy. The discouraging results of non-RAI treatment methods in these patients led the researchers to seek out new methods to reestablish RAI uptake. ${ }^{3}$ For this purpose retinoic acids (RAs) were one of the first agents to be used for their redifferentiation induction properties. Simon et al. showed an increase in RAI uptake in 8 of 20 patients following treatment with $1.5 \mathrm{mg} / \mathrm{kg}$ of RA. ${ }^{4}$ However, other studies did not support these initially encouraging results. ${ }^{5}$ Therefore, new innovative methods are needed to reestablish radioiodine uptake in the dedifferentiated thyroid tumors. 5-azacytidine, a DNA methyltransferase inhibitor, is a promising agent for this purpose, which prevents the methylation of certain genes and modulates their activities. ${ }^{6}$ Initial experimental studies are encouraging. Venkataraman et al. was able to restore hNIS mRNA expression in four cell lines and iodide transport in two cell lines following treatment with 5-azacytidine. ${ }^{7}$ Trichostatin A, which is a histone deacetylase inhibitor, was also tried to reestablish radioiodine uptake. Zarnegar et al. showed an increase in mean NIS gene expression following treatment with trichostatin A. ${ }^{8}$ After these successful preclinical studies, a histone deacetylase inhibitor, suberoylanilide hydroxamic acid (SAHA), was used orally and established radioiodine uptake in 1 of 3 patients. ${ }^{9}$ In the literature, the studies with these agents are limited and the differential effects of these drugs on thyroid-specific genes in different tumoral and normal thyroid cell lines need further clarification. In this study, we investigated the role of these gene modulators on the mRNA expressions of NIS, Tg, TPO, TSH-R, and RAI uptake in cancer and normal thyroid cell lines.

\section{MATERIAL AND METHODS}

\section{Cell Lines and Chemicals}

B-CPAP is a tumoral cell line derived from a 76year-old patient with metastatic papillary cancer and was obtained from DSMZ (Deutsche Sammlung von Mikroorganismen und Zellkulturen $\mathrm{GmBH}$
[German Collection of Microorganisms and Cell Cultures; Braunschweig, Germany). ${ }^{10}$ Nthy-ori 3-1 is a normal thyroid follicular epithelial cell line, immortalized by SV-40 and obtained from ECACC (European Collection of Cell Cultures; Wiltshire, United Kingdom). ${ }^{11}$ Both cell lines were grown in RPMI 1640 medium supplemented with $2 \mathrm{mM}$ of glutamine and $10 \%$ fetal bovine serum (FBS) at $37^{\circ} \mathrm{C}$ and $5 \% \mathrm{CO}_{2}$. The reagents used, 5-Aza, TSA, ATRA, RPMI 1640, N,N dimetilformamid (DMF), FBS, penisilin-streptomisin and L-glutamine, were purchased from Sigma Chemical Company (St. Louis, MO) and recombinant TSH (rTSH); (Thyrogen ${ }^{\circledR}$ ) was obtained from the Genzyme corporation (Cambridge, MA).

\section{MTT (Methyl-Thiazol-Tetrazolium) Test}

Cells were placed in 96-well plates at a density of 8000 cells/well for 24 hours and 2000 cells/well for 48- and 72-hour incubations. Following treatment with different doses of TSA (10-50 ng/mL), 5-Aza (1-50 $\mu \mathrm{M})$, and ATRA (0,01-100 $\mu \mathrm{M})$ for 24,48 , and 72 hours, the media were removed and the cells were incubated with MTT (concentration of $1 \mathrm{mg} / \mathrm{ml}$ ) for 4 hours. Viable cells convert yellow-colored MTT to violet-colored formazan crystals by using the mitochondrial succinate dehydrogenase. These formazan crystals were dissolved in dimethyl sulfoxide (DMSO; $\mathrm{pH}, 4.7$ ), prepared after being dissolved in $45 \%$ DMF, and the amount of formazan crystals reflecting cellular growth and viability was determined quantitatively by absorbance measurements in spectrophotometric assays. The sublethal maximum doses for every gene modulator were determined according to the MTT results. rTSH levels were determined by the maximum effective dose defined $(100 \mathrm{ng} / \mathrm{mL})$ in the literature and applied for 4 and 9 days. ${ }^{12}$

\section{RNA Isolation and RT-PCR (Real-Time-Polymerase Chain Reaction)}

After treatment with gene modulators, RNA isolation from the cells were made by a Qiagen RNAeasy kit (Qiagen Inc., Valencia, CA), using the "Spin" protocol. For RT-PCR, a Quantitech RT-PCR kit obtained from Qiagen Inc. and primers and probes obtained from the Motek company (Istanbul, Turkey) were used. The dyes used in the probes were black hole and FAM. RTPCR was done in an ICycler thermal cycler (BioRad; Hercules, CA) compatible with the optic detection system, iCycler iQ (Biorad). Gene ex- 
Table 1. Basal Levels of Thyroid Specific Gene Expressions

\begin{tabular}{|c|c|c|c|c|c|c|c|c|}
\hline Gene & $\begin{array}{c}B^{\mathrm{b}} \\
T S H-R\end{array}$ & $\begin{array}{c}N^{\mathrm{a}} \\
T S H-R\end{array}$ & $\begin{array}{c}B^{\mathrm{b}} \\
T P O\end{array}$ & $\begin{array}{c}N^{\mathrm{a}} \\
T P O\end{array}$ & $\begin{array}{l}B^{\mathrm{b}} \\
T g\end{array}$ & $\begin{array}{l}N^{\mathrm{a}} \\
T g\end{array}$ & $\begin{array}{c}B^{\mathrm{b}} \\
N I S\end{array}$ & $\begin{array}{c}N^{\mathrm{a}} \\
N I S\end{array}$ \\
\hline Target $\mathrm{Ct}$ & 31.9 & 29.0 & 29.0 & 35.5 & 42.0 & 27.2 & 32.6 & 31.3 \\
\hline GAPDH Ct & 11.3 & 10.1 & 11.5 & 11.6 & 11.9 & 10.6 & 11.7 & 11.0 \\
\hline Target/GAPDH & 2.83 & 2.90 & 2.52 & 3.06 & 3.52 & 2.56 & 2.78 & 2.84 \\
\hline
\end{tabular}

TSH-R, thyroid-stimulating hormone receptor; TPO, thyroid peroxidase; Tg, thyroglobulin; NIS, Na/I symporter; Ct, value of cycles cells require to reach threshold gene expression; GADPH, glyceraldehyde-3-phosphate dehydrogenase.

aNthy-ori 3-1.

${ }^{\mathrm{b}} \mathrm{B}-\mathrm{CPAP}$.

pression studies were repeated three times and specific gene expressions were normalized with GADPH (glyceraldehyde-3-phosphate dehydrogenase) mRNA. Expression results were calculated in terms of $\mathrm{Ct}$ values and relative gene expression. $\mathrm{Ct}$ values represent the value of cycles that the cells require to reach the threshold gene expression, and these values were inversely correlated with gene expression. The relative gene expression was calculated with the formula, $\mathrm{Ct}=$

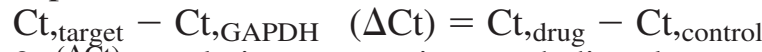
$2^{-(\Delta \mathrm{Ct})}=$ relative expression, and directly proportional to the gene expression.

\section{Iodide Uptake}

Two (2) $\times 10^{5}$ cells/well were plated in 24-well plates and cultured with RPMI 1640 medium containing 10\% FBS. Following treatment, for 3 days the cells were, washed with Hanks' balanced salt solution (HBSS) and incubated for 1 hour at $37^{\circ} \mathrm{C}$ with $500 \mathrm{~mL}$ of HBSS containing $0.1 \mathrm{mCi}$ of carrier-free $\mathrm{NaI}^{125}$ (Cisbio; Bagnols/Cèze Cedex, France) and $10 \mathrm{mM}$ of NaI. For perchlorate inhibition studies, $\mathrm{NaClO}_{4}$ was added as a solution in HBSS, with a final concentration of $30 \mu \mathrm{M}$, im- mediately following the addition of radiolabeled iodine. Reactions were rapidly terminated by removing the radioactive HBSS and washing the cells twice with ice-cold HBSS. The cells were then extracted following solubilization with $90 \%$ ethanol for 30 minutes, and radioactivity was measured by a gamma counter. ${ }^{13,14}$ The RAI uptake values were given in values presenting the specific uptake that can be inhibited by $\mathrm{NaClO}_{4}$.

\section{RESULTS}

\section{MTT Test}

In the MTT tests, TSA and ATRA showed similar death rates in both cells, but 5-Aza showed toxicity only in the B-CPAP cell line but not in Nthy-ori 3-1 (Mann-Whitney U test; $p=0.029$ ). According to the MTT results, cells were treated with the sublethal doses of $10 \mathrm{ng} / \mathrm{mL}$ for TSA, 5 $\mu \mathrm{M}$ for 5-Aza, and $1 \mu \mathrm{M}$ for ATRA.

\section{RT-PCR and RAI Uptake}

Basal thyroid-specific gene expressions are given in Table 1. In both cell lines, basal NIS and TSH-

Table 2. Twenty-four (24), 48-, and 72-Hour Relative Expressions of Thyroid Specific Genes Are Shown After a 5- $\mu$ M 5-azacytidine Application

\begin{tabular}{lcccccccc}
\hline & Nthy-ori & Nthy-ori & Nthy-ori & Nthy-ori & BCPAP & BCPAP & BCPAP & BCPAP \\
& TPO & NIS & Tg & TSH-R & TPO & NIS & Tg & TSH-R \\
\hline Control & 1.00 & 1.00 & 1.00 & 1.00 & 1.00 & 1.00 & 1.00 & 1.00 \\
AZA 24 hours & 2.14 & 0.57 & 0.38 & 4.29 & 0.35 & 0.31 & 1.15 & 1.15 \\
AZA 48 hours & 0.57 & 1.62 & 0.71 & 1.32 & 0.27 & 0.31 & 0.71 & 6.06 \\
AZA 72 hours & 0.47 & 0.81 & 1.15 & 0.87 & 0.38 & 0.31 & 0.62 & 2.46 \\
\hline
\end{tabular}

TPO, thyroid peroxidase; NIS, Na/I symporter; Tg, thyroglobulin; TSH-R, thyroid-stimulating hormone receptor; AZA, azacytidine. 


\begin{tabular}{|c|c|c|c|c|c|c|c|c|}
\hline & $\begin{array}{c}\text { Nthy-ori } \\
\text { TPO }\end{array}$ & $\begin{array}{c}\text { Nthy-ori } \\
\text { NIS }\end{array}$ & $\begin{array}{c}\text { Nthy-ori } \\
T g\end{array}$ & $\begin{array}{c}\text { Nthy-ori } \\
\text { TSH-R }\end{array}$ & $\begin{array}{c}\text { ВСРAP } \\
T P O\end{array}$ & $\begin{array}{c}\text { ВCPAP } \\
\text { NIS }\end{array}$ & $\begin{array}{c}B C P A P \\
T g\end{array}$ & $\begin{array}{c}B C P A P \\
T S H-R\end{array}$ \\
\hline Control & 1.00 & 1.00 & 1.00 & 1.00 & 1.00 & 1.00 & 1.00 & 1.00 \\
\hline TSA 24 hours & 1.07 & 0.57 & 0.76 & 0.71 & 0.54 & 0.47 & 0.81 & 0.38 \\
\hline TSA 48 hours & 0.33 & 1.87 & 0.31 & 0.76 & 1.07 & 0.23 & 0.11 & 1.00 \\
\hline TSA 72 hours & 1.15 & 0.76 & 1.23 & 3.03 & 0.93 & 0.44 & 0.57 & 1.41 \\
\hline
\end{tabular}

TPO, thyroid peroxidase; NIS, Na/I symporter; Tg, thyroglobulin; TSH-R, thyroid-stimulating hormone receptor; TSA, trichostatin-A.

R mRNA levels were similar, but basal TPO expression was higher in the B-CPAP cell line and Tg expression was higher in the Nthy-ori 3-1 cell line. 5-Aza increased TSH-R mRNA expression in both of the cell lines and decreased TPO, NIS, and $\mathrm{Tg}$ mRNA levels in the cancer cell line. In the normal thyroid cell line, 5-Aza increased TPO mRNA levels 2-fold and made no differences in NIS and Tg mRNA levels (Table 2). TSA treatment repressed NIS and Tg mRNA levels and made no changes on other thyroid-specific genes that were investigated in the cancer cell line. In the normal thyroid cell line, TSA increased TSH$\mathrm{R}$ mRNA levels in 72 hours and created no important differences in other genes (Table 3). ATRA repressed the TSH-R mRNA levels in the normal thyroid cell line and increased the TPO and Tg mRNA levels slightly in both cell lines. Furthermore, in the short-term treatment, ATRA repressed NIS gene expression slightly, but in the long term, this repression turned to basal levels (Table 4). TSA and ATRA did not make any significant changes in RAI uptake in the cancer cell line. 5-Aza slightly increased RAI uptake in the cancer cell line, which was not statistically significant $(p>0.05)$. rTSH increased RAI uptake significantly (Fig. 1). In the normal thyroid cell line, TSA and ATRA decreased RAI uptake (to 1/10 and $1 / 2$, respectively) but 5-Aza and rTSH increased RAI uptake significantly (2- and 4-fold, respectively) (Fig. 2) ( $p=0.011$ and $p<0.0001)$.

\section{DISCUSSION}

The treatment options for the patients who had residual thyroid cancer, but negative I-131 scintigraphy, were limited. Several authors suggested at least one empirical high-dose RAI treatment, and if there was an RAI uptake in posttherapy images and/or a decrease in Tg levels, then further therapy with RAI would be warranted. ${ }^{15}$ If there was no response to empirical RAI therapy, chemotherapy and/or radiotherapy could be tried. These treatments were used only for palliative purposes owing to the low success and high side-effect rate. In the literature, adriamycin treatment was reported to have a $31 \%$ and $37 \%$ partial response rate. ${ }^{16,17}$ These inadequate response rates led the researchers to seek out new methods to reestablish RAI uptake in tumor cells. RA was one of the first agents to be tried in the

Table 4. Twenty-four (24), 48-, and 72-Hour Relative Expressions of Thyroid Specific Genes Are Shown After an Application of $1 \mu \mathrm{M}$ of All-Trans Retinoic Acid

\begin{tabular}{lcccccccc}
\hline & Nthy-ori & Nthy-ori & Nthy-ori & Nthy-ori & BCPAP & BCPAP & BCPAP & BCPAP \\
& TPO & NIS & Tg & TSH-R & TPO & NIS & Tg & TSH-R \\
\hline Control & 1.00 & 1.00 & 1.00 & 1.00 & 1.00 & 1.00 & 1.00 \\
ATRA 24 hours & 2.46 & 0.80 & 1.74 & 0.21 & 2.29 & 0.65 & 2.80 & 1.00 \\
ATRA 48 hours & 1.74 & 1.50 & 2.14 & 0.28 & 2.14 & 0.65 & 1.14 & 0.75 \\
ATRA 72 hours & 1.62 & 0.60 & 1.86 & 0.18 & 1.14 & 1.14 & 0.812 \\
\hline
\end{tabular}

TPO, thyroid peroxidase; NIS, Na/I symporter; Tg, thyroglobulin; TSH-R, thyroid-stimulating hormone receptor; ATRA, alltrans retinoic acid. 

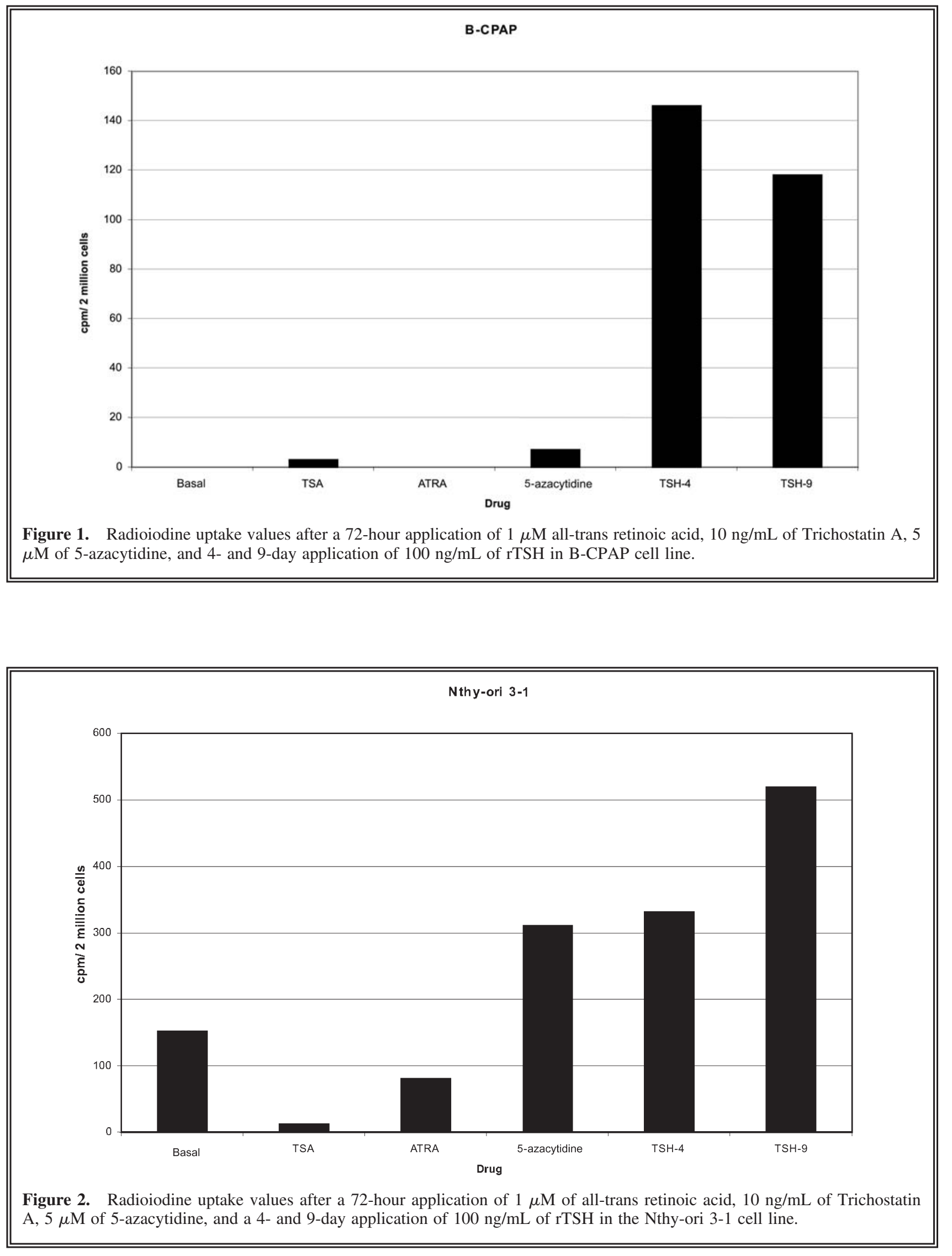
reinduction of RAI uptake owing to its redifferentiation effect.

In preclinical studies, Schmutzler et al. showed an increase in NIS gene expression in FTC-133 and 238 follicular thyroid tumoral cell lines but no change in anaplastic thyroid cell lines HTh74 and C643 after treatment with $1 \mu \mathrm{M}$ all-trans retinoic acid. In the same study, retinoic acid application caused a decrease in NIS mRNA levels and iodide uptake (38\%) in normal rat thyroid cell line, FTRL$5 .{ }^{18}$ In the Nthy-ori 3-1 cell line, ATRA repressed TSH-R mRNA levels and showed an increase in TPO and Tg mRNA levels in both cell lines (maximum, 2.5-fold). This increase in Tg mRNA levels was also detected by Kurebayashi et al. in a recurrent thyoid cancer cell line (KTC-1). ${ }^{19}$ In the NIS gene, ATRA treatment caused a minimal suppression in short-term incubation, but returned to normal in both cell lines (Table 4). In Nthy-ori 3-1 cells, ATRA supressed RAI uptake and had no significant effect on B-CPAP cell lines. The supressive effect of ATRA in normal cell lines is compatible with the results reported by Schmutzler et al. in FTRL-5. ${ }^{18}$ This finding supports the idea that ATRA shows different effects in the normal and tumoral thyroid cell lines.

Haugen et al. showed that the receptors responsible for the action of RA, namely $\operatorname{RAR} \beta$ and $\operatorname{RXR} \gamma$, were different in tumoral cell lines and the RXR $\gamma$ form was not found in normal cell lines. As expected, RA treatment was found to be only effective in tumors expressing $\operatorname{RAR} \beta$ and $\operatorname{RXR} \gamma \cdot{ }^{20}$ This literature finding may explain the ineffectiveness of ATRA in the B-CPAP cell line, which we used in our study. The differential effects of RA on different cell lines and genes need to be confirmed by further molecular studies on both receptor and gene bases.

In our study, 5-azacytidine showed toxicity only in the B-CPAP cell lines, but not in the Nthyori 3-1 cell line, according to the MTT test results (Mann-Whitney U test; $p=0.029$ ). In their study, Schmelz et al. showed an increase in apoptotic p21WAF gene expression following treatment with the methylation inhibitor, 5-Aza-2'-deoksysitidin, and this leads to death in apoptotic myeloid leukemia cells. ${ }^{21}$ Szyf found that, contrary to tumoral cells, normal cell lines do not show death after the inhibition of the methylation enzyme. According to the authors, this was related with the absence of methylation in tumor suppressor genes (p21). ${ }^{22}$

These literature results suggested a possible inactive tumor suppressor gene in B-CPAP leading to death after activation by methylation inhibition, which may explain our MTT test results. In our study, 5-Aza increased TSH-R mRNA expressions in both cell lines (Table 2). This finding is compatible with the findings by Xing et al., who showed a methylation of TSH-R in 23 of 39 (59\%) papillary thyroid carcinomas and 7 of 15 $(47 \%)$ follicular thyroid carcinomas. ${ }^{23}$ After binding to its receptor, TSH starts a signaling pathway, which increases the expression of thyroid-specific genes and RAI uptake. During the dedifferentiation process, the tumoral cells lose their TSH-R and grow independently. ${ }^{24}$ TSH-R is thought to have functions other than the transmission of TSH signal. Hoelting et al. found that in most of the metastatic aggressive follicular cancer cells, TSH-R was absent, and after transfection of this receptor, the cells showed a decrease in invasion and growth capacity. The authors concluded that the presence of TSH-R also functions in the control of cell growth. ${ }^{25}$ In our study, we have shown an increase in TSH-R by 5-Aza in the tumoral cell line. 5-Aza, by reinducing the expression of TSH-R in the tumoral cell line, may increase the effect of TSH, which is crucial for RAI uptake but may also control cell growth. In our study, 5-Aza increased TPO mRNA levels 2-fold and made no significant differences in NIS and Tg mRNA levels in the normal thyroid cell line. The increase in TPO mRNA supported the increased RAI uptake in the normal thyroid cell line $(p=0.011)$.

To our knowledge, this is the first study to show the effects of 5-Aza in normal thyroid cells and must be supported by further studies. 5-Aza decreased TPO, NIS, and Tg mRNA levels in the cancer cell line but slightly increased RAI uptake in the cancer cell line, which was not statistically significant $(p>0.05)$. These results were contrary to the results of Venkataraman et al. ${ }^{7}$ showing an increase in NIS mRNA and RAI uptake in thyroid cancer cell lines following treatment with 5-Aza. This difference can be a result of different thyroid cell lines, which may differ in their methylation characteristics.

We chose the B-CPAP cell line for our experimental model, which is a relatively differentiated cell line, as determined by better basal thyroid-specific gene expression levels ${ }^{10,25-27}$ (Table 1 ) and it fits to the clinical scenario (relatively differentiated and preserved its genetic information but lost its I-131 accumulation capacity). The cell lines with different genetic characteristics may respond differently to demethylating agents. 
Moreover, as shown by Venkatarman et al., the effect of these agents were not solely dependent on gene expression levels, but also to the activities of transcription factors, such as thyroid transcription factor- $1 .^{7}$ So, the slight increase in RAI uptake in our study can be attributed to the effect of 5-Aza on these transcription factors.

For a further clarification of this issue, we suggest an analysis of the tumor cell lines for their methylation status at each gene level prior to a treatment with demethylating agents. In our study, TSA treatment repressed NIS and Tg mRNA levels and made no changes to other thyroid-specific genes that were investigated in the cancer cell line. In the normal thyroid cell line, TSA increased TSH-R mRNA levels in 72 hours and created no important differences in other genes (Table 3). TSA did not make any changes in RAI uptake in the cancer cell line. In the normal thyroid cell line, TSA decreased RAI uptake.

Contrary to our results, Zarnegar et al. found that following treatment with TSA, NIS expression was increased 107- and 217-fold in papillary, 39- and 58-fold in hurthle, and 459- and 781fold in follicular thyroid cancers. ${ }^{8}$

In the literature, all of the studies with TSA were done with dedifferentiated cancers. The ineffectiveness of TSA in our study and the decrease in RAI uptake in normal cells also suggested in inverse relation with differentiation status as proposed with RA. In our study, the only benefit from TSA was a slight increase in TSH$\mathrm{R}$ expression, which was less significant than 5Aza. As expected, rTSH increased RAI uptake in both cell lines (Figs. 1 and 2). The application of rTSH, especially with the agents that increase TSH-R, must be further evaluated for a possible synergistic effect.

\section{CONCLUSIONS}

We have shown an increase in TSH-R gene expression and radioiodine uptake with 5-Aza. Further in vitro and in vivo studies are needed to support our findings and the potential clinical use of this agent.

\section{ACKNOWLEDGMENT}

This study was supported by grants: the Scientific and Technological Research Council of Turkey Fund no. SBAG-2771; Hacettepe Uni- versity Research Fund no. 04 D04 101 001; Turkish Academy of Science Fund no. OU/TUBAGEBIP-2003-16.

\section{REFERENCES}

1. American Association of Clinical Endocrinologists, American Association of Endocrine Surgeons. AACE/ AAES medical/surgical guidelines for clinical practice: Management of thyroid carcinoma. Endocr Pract 2001;3:202.

2. Mazzaferri EII, Kloos RT. Current approaches to primary therapy for papillary and follicular thyroid cancer. J Clin Endocrinol Metab 2001;86:1447.

3. Haugen BR. Management of the patient with progressive radioiodine nonresponsive disease. Semin Surg Oncol 1999;16(1):34.

4. Simon D, Koehrle J, Reiners C, et al. Redifferentiation therapy with retinoids: Therapeutic option for advanced follicular and papillary thyroid carcinoma. W J Surg 1998;22(6):569.

5. Courbon F, Zerdoud S, Bastie D, et al. Defective efficacy of retinoic acid treatment in patients with metastatic thyroid carcinoma. Thyroid 2006;16(10):1025.

6. Noyer-Weidner M, Trautner TA. Methylation of DNA in prokaryotes. EXS 1993;64:39.

7. Venkataraman GM, Yatin M, Marcinek R, et al. Restoration of iodide uptake in dedifferentiated thyroid carcinoma: Relationship to human $\mathrm{Na}^{+} / \mathrm{I}$-symporter gene methylation status. J Clin Endocrinol Metab 1999;84:2449.

8. Zarnegar R, Brunaud L, Kanauchi H, et al. Increasing the effectiveness of radioactive iodine therapy in the treatment of thyroid cancer using Trichostatin A, a histone deacetylase inhibitor. Surgery 2002;132: 984.

9. Tuttle M, Kelly WK, Robbins R. SAHA, an oral histone deacetylase inhibitor, slows clinical disease progression in advanced thyroid cancer. [abstr.] Thyroid 2004; 14:683.

10. Fabien N, Fusco A, Santoro M, et al. Description of a human papillary thyroid carcinoma cell line: Morphologic study and expression of tumoral markers. Cancer 1994;73:2206.

11. Lemoine NR, Mayall ES, Jones T, et al. Characterisation of human thyroid epithelial cells immortalised in vitro by simian virus 40 DNA transfection. Br J Cancer 1989;60:897.

12. Grossmann M, Szkudlinski MW, Tropea JE, et al. Expression of human thyrotropin in cell lines with different glycosylation patterns combined with mutagenesis of specific glycosylation sites: Characterization of a novel role for the oligosaccharides in the in vitro and in vivo bioactivity. J Biol Chem 1995;270(49):29378.

13. Nakamoto Y, Saga T, Misaki T, et al. Establishment and characterization of a breast cancer cell line expressing $\mathrm{Na} / \mathrm{I}$ symporters for radioiodide concentrator gene therapy. J Nucl Med 2000;41:1898. 
14. Weiss SJ, Philipp NJ, Grollmann EF. Iodine transport in a continuous line of cultured cells from rat thyroid. Endocrinology 1984;114:1090.

15. Ringel MD, Ladenson PW. Controversies in the followup and management of well-differentiated thyroid cancer. Endocr Rel Cancer 2004;11:97.

16. Frei E, Luce JK, Middleman E. Clinical trials of Adriamycin. In: Carter SK, DiMarco A, Ghione M, et al., (eds.) International Symposium on Adriamycin. BerlinNew York: Springer, 1972;152.

17. Kolaric K, Maracic Z, Nola P, et al. Modified administration schedule of adriamycin in solid tumors. Z Krebsforsch Klin Onkol Cancer Res Clin Oncol 1977;88:255.

18. Schmutzler C, Winzer R, Meissner-Weigl, J et al. Retinoic acid increases sodium/iodide symporter mRNA levels in human thyroid cancer cell lines and suppresses expression of functional symporter in nontransformed FRTL-5 rat thyroid cells. Biochem Biophys Res Commun 1997;26;240(3):832.

19. Kurebayashi J, Tanaka K, Otsuki T, et al. Alltrans-retinoic acid modulates expression levels of thyroglobulin and cytokines in a new human poorly differentiated papillary thyroid carcinoma cell line, KTC-1. J Clin Endocrinol Metab 2000;85(8): 2889.

20. Haugen BR, Larson LL, Pugazhenthi, et al. Retinoic acid and retinoid $\mathrm{X}$ receptors are differentially ex- pressed in thyroid cancer and thyroid carcinoma cell lines, and predict response to treatment with retinoids. J Clin Endocrinol Metab 2004;89:272.

21. Schmelz K, Wagner M, Dorken B, et al. 5-Aza-2'-deoxycytidine induces $\mathrm{p} 21 \mathrm{WAF}$ expression by demethylation of p73 leading to p53-independent apoptosis in myeloid leukemia. Int J Cancer 2005;114:683.

22. Szyf M. Targeting DNA methylase in cancer. Cancer Metast Rev 1998;17:219.

23. Xing M, Usadel H, Cohen Y, et al. Methylation of the thyroid-stimulating hormone receptor gene in epithelial thyroid tumors: A marker of malignancy and a cause of gene silencing. Cancer Res May 2003;63(9):2316.

24. Brabant G, Maenhaut C, Kohrle J, et al. Human thyrotropin receptor gene: Expression in thyroid tumors and correlation to markers of thyroid differentiation and dedifferentiation. Mol Cell Endocrinol 1991;82:R7.

25. Hoelting T, Duh QY, Clark OH, et al. Transfection of follicular thyroid gland carcinoma cells with human TSH receptor changes growth, invasion, and adhesion Langenbecks Arch Chir Suppl Kongress 1999;115:281.

26. Petrich T, Knapp WH, Potter E. Functional activity of human sodium/iodide symporter in tumor cell lines. Nuklearmedizin 2003;42(1):15.

27. Arturi F, Russo D, Bidart JM, et al. Expression pattern of the pendrin and sodium/iodide symporter genes in human thyroid carcinoma cell lines and human thyroid tumors. Eur J Endocrinol 2001;145(2):129. 